\title{
Letter to the editor: COVID-19 and all-cause excess mortality in Iran in spring 2020
}

\author{
Hooman Tadbiri ${ }^{1}$, Maziar Moradi-Lakeh*2(D), Mohsen Naghavi ${ }^{3}$ \\ Received: 9 Aug $2020 \quad$ Published: 22 Sep 2020
}

Conflicts of Interest: None declared

Funding: No funding

*This work has been published under CC BY-NC-SA 1.0 license.

Copyright $\odot$ Iran University of Medical Sciences

Cite this article as: Tadbiri H, Moradi-Lakeh M, Naghavi M. Letter to the editor: COVID-19 and all-cause excess mortality in Iran in spring 2020. Med J Islam Repub Iran. 2020 (22 Sep);34:125. https://doi.org/10.47176/mjiri.34.125

Several types of data, including, but not limited to, the number of confirmed cases, hospitalizations, reported confirmed COVID-19 deaths, excess deaths, and representative prevalence surveys are being suggested to understand the extent and spread of COVID-19. These types of data are usually correlated, but each of them has its strengths and limitations. For instance, both the number of confirmed cases and reported confirmed COVID-19 deaths are likely to be underestimated. Excess deaths (ie, the number of deaths observed above the expected baseline) are "the best indicator of the mortality impacts of the pandemic" according to a National Academies of Science, Engineering, and Medicine (NASEM) review (1). We previously reported an estimate for the excess deaths in Iran at national and provincial levels in the latest fall and winter using the vital statistics data from the National Organization for Civil Registration (NOCR). The estimated number of excess deaths at the national level in the winter of 1398 SH (December 22, 2019 - March 19, 2020) was remarkably higher than the reported confirmed COVID-19 deaths in that period, and our estimations at provincial level were highly correlated with the official provincial number of COVID-19 cases. We concluded most of the excess deaths are related to COVID-19 (2).

Corresponding author: Dr Maziar Moradi-Lakeh, moradilakeh.m@iums.ac.ir

1. Johns Hopkins Bloomberg School of Public Health, Baltimore, MD, USA

2. Preventive Medicine and Public Health Research Center, Psychosocial Health Research Institute, Iran University of Medical Sciences, Tehran, Iran

3. Institute for Health Metrics and Evaluation, University of Washington, Seattle, WA USA
The Ministry of Health and Medical Education (MoHME) in Iran reports the number of confirmed cases and confirmed COVID-19 deaths at the national level. MoHME reported 184177 confirmed cases of COVID-19 and 8223 deaths with laboratory-confirmed SARS-CoV-2 during the spring of 1399 SH (March 20, 2020 - June 20, 2020) $(3,4)$. The number of confirmed cases and the number of confirmed deaths of COVID-19 at the provincial level are not publicly available.

Recently, NOCR has reported 110384 registered deaths during the spring of $1399 \mathrm{SH}$, which is 18642 deaths higher than the same season in the previous year. As illustrated in Figure 1, the number of deaths in the spring of $1399 \mathrm{SH}$ in all provinces were higher than the previous year. Furthermore, there were 4597 more deaths in the spring of 1399 SH compared to the winter of $1398 \mathrm{SH}$ (5). This is not compatible with the seasonal pattern of deaths in nonequatorial countries, which usually have a lower number of deaths in the warmer seasons $(6,7)$, and this is the first spring in Iran since the data are publicly available (1393 SH, 2014 Gregorian calendar) that has a higher number of deaths compared to its past winter. For instance, the number of registered deaths in the winter of $1397 \mathrm{SH}$ (December 22, 2018 - March 20, 2019) and the spring of 1398 SH (March 21,

$\uparrow$ What is "already known" in this topic:

Our previous study showed that the number of excess deaths in the winter of 1398 is much higher than the confirmed officially reported COVID-19 cases.

$\rightarrow$ What this article adds:

Based on the new analysis, we estimated 16512 (95\% CI: 12 333-20 691) excess deaths for Spring of 1399.

Provincial excess deaths are correlated with the rates of COVID19 hospital admissions. We think most of these excess deaths are COVID-19-related. 


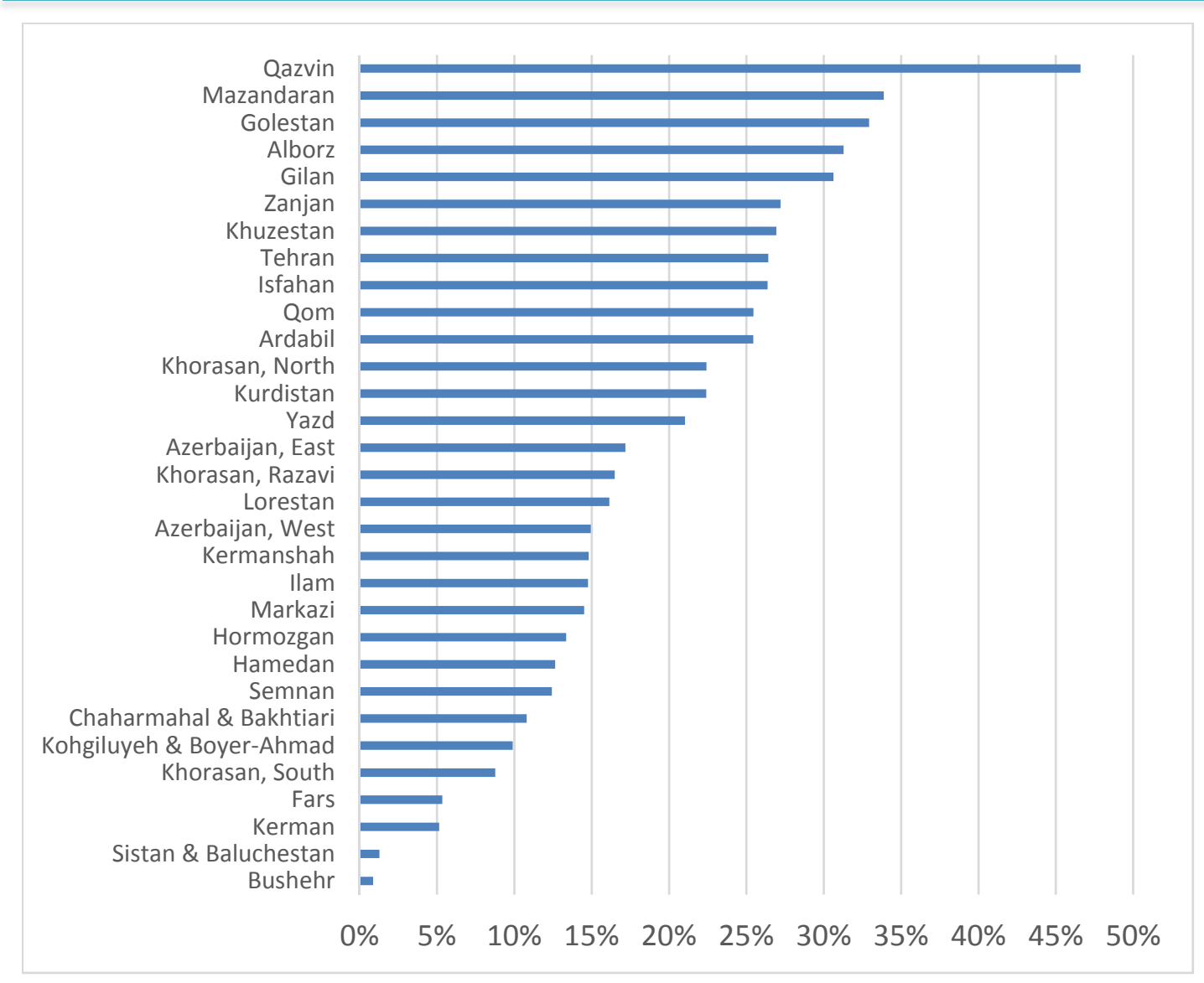

Fig. 1. Increase in the number of registered deaths in the spring of $1399 \mathrm{SH}$ (March 20, 2020 - June 20,2020) compared to the registered deaths in the spring of $1398 \mathrm{SH}$ (March 21, 2019 - June 21, 2019), as the percentage of registered deaths of the spring of $1398 \mathrm{SH}$, by province

2019 - June 21, 2020) were 101210 and 91 742, respectively.

We applied the same statistical approach that was explained in detail in our previous report (2) to account for historical trend and seasonal pattern of all-cause deaths in estimation of expected number of deaths; we estimated 16 512 (95\% CI: 12 333-20 691) excess deaths at the national level in the spring of $1399 \mathrm{SH}$. This number is again remarkably higher than the official number of COVID-19 deaths in the corresponding period, and it is also greatly increased compared to the estimated 5180 (95\% CI: 10239337) excess deaths of the winter of 1398 SH (Fig. 2). The results of our analyses by province are presented in Table 1.

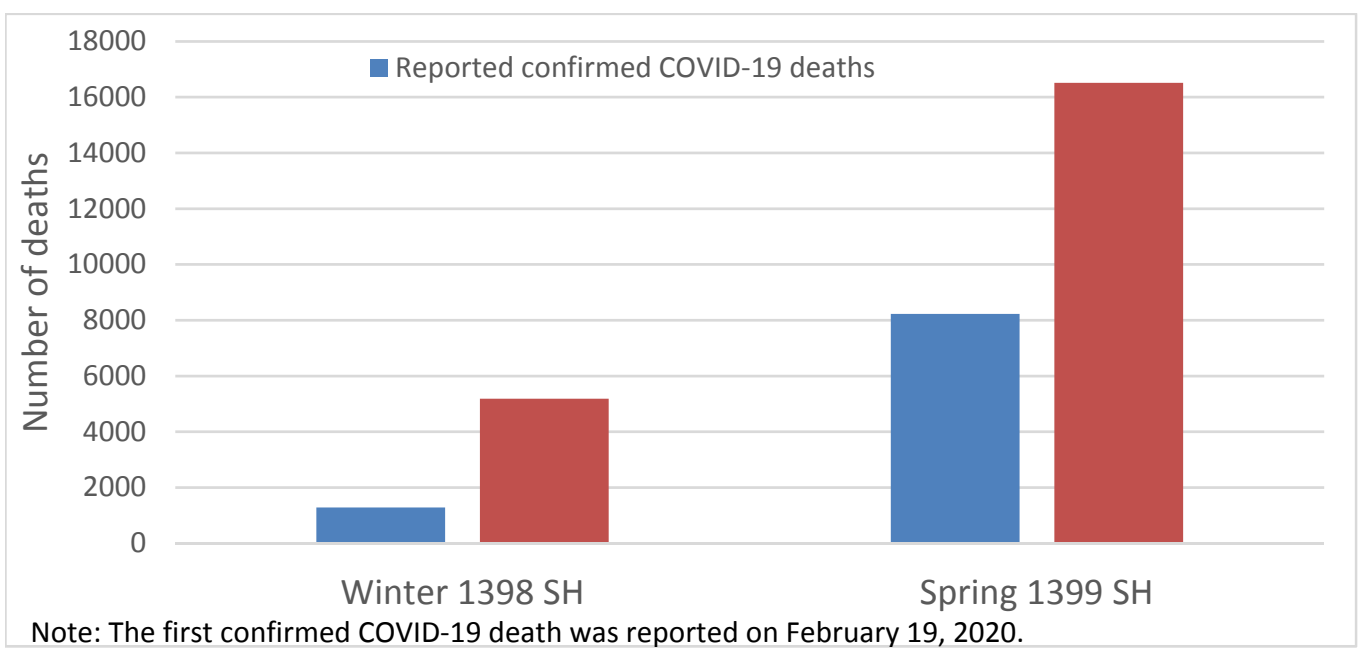

Fig. 2. Number of reported confirmed COVID-19 deaths in Iran in the winter of 1398 SH (December 22, 2019 - March 19, 2020) and the spring of 1399 SH (March 20, 2020 - June 20, 2020) compared to the estimated excess deaths 
Table 1. Estimated excess deaths in the spring of 1399 SH (March 20, 2020 - June 20, 2020) by province

\begin{tabular}{|c|c|c|c|}
\hline \multirow[t]{2}{*}{ Province } & \multirow[t]{2}{*}{ Excess deaths } & \multicolumn{2}{|c|}{$95 \%$ confidence interval } \\
\hline & & Lower bound & Upper bound \\
\hline Alborz & $638(18.96 \%)$ & $348(10.35 \%)$ & $928(27.58 \%)$ \\
\hline Ardabil & $400(19.00 \%)$ & $222(10.55 \%)$ & $578(27.45 \%)$ \\
\hline Azerbaijan, East & $1,066(16.44 \%)$ & $787(12.13 \%)$ & $1,346(20.75 \%)$ \\
\hline Azerbaijan, West & $589(13.57 \%)$ & $373(8.60 \%)$ & $804(18.53 \%)$ \\
\hline Bushehr & $-5(-0.49 \%)$ & $-112(-10.88 \%)$ & $102(9.90 \%)$ \\
\hline Chaharmahal and Bakhtiari & $74(7.04 \%)$ & $-30(-2.84 \%)$ & $179(16.92 \%)$ \\
\hline Fars & $108(1.95 \%)$ & $-158(-2.85 \%)$ & $374(6.74 \%)$ \\
\hline Gilan & $1,104(21.00 \%)$ & $892(16.96 \%)$ & $1,317(25.04 \%)$ \\
\hline Golestan & $678(23.72 \%)$ & $585(20.47 \%)$ & $771(26.98 \%)$ \\
\hline Hamedan & $391(13.29 \%)$ & $109(3.71 \%)$ & $672(22.87 \%)$ \\
\hline Hormozgan & $136(8.06 \%)$ & $-35(-2.09 \%)$ & $306(18.22 \%)$ \\
\hline Ilam & $61(8.80 \%)$ & $-14(-1.99 \%)$ & $135(19.58 \%)$ \\
\hline Isfahan & $1,444(20.13 \%)$ & $948(13.22 \%)$ & $1,940(27.05 \%)$ \\
\hline Kerman & $-23(-0.65 \%)$ & $-341(-9.79 \%)$ & $295(8.49 \%)$ \\
\hline Kermanshah & $348(11.10 \%)$ & $40(1.28 \%)$ & $655(20.92 \%)$ \\
\hline Khorasan, North & $180(14.01 \%)$ & $65(5.04 \%)$ & $295(22.99 \%)$ \\
\hline Khorasan, Razavi & $972(11.21 \%)$ & $544(6.27 \%)$ & $1,400(16.15 \%)$ \\
\hline Khorasan, South & $132(12.48 \%)$ & $-6(-0.52 \%)$ & $269(25.48 \%)$ \\
\hline Khuzestan & $1,299(20.31 \%)$ & $905(14.16 \%)$ & $1,693(26.47 \%)$ \\
\hline Kohgiluyeh and Boyer-Ahmad & $69(9.17 \%)$ & $-21(-2.77 \%)$ & $159(21.10 \%)$ \\
\hline Kurdistan & $454(20.36 \%)$ & $280(12.55 \%)$ & $628(28.16 \%)$ \\
\hline Lorestan & $395(15.11 \%)$ & $169(6.48 \%)$ & $620(23.75 \%)$ \\
\hline Markazi & $284(12.80 \%)$ & $134(6.04 \%)$ & $433(19.55 \%)$ \\
\hline Mazandaran & $1,338(24.56 \%)$ & $1,109(20.36 \%)$ & $1,567(28.76 \%)$ \\
\hline Qazvin & $594(28.75 \%)$ & $457(22.10 \%)$ & $732(35.39 \%)$ \\
\hline Qom & $342(18.74 \%)$ & $272(14.91 \%)$ & $412(22.58 \%)$ \\
\hline Semnan & $124(12.73 \%)$ & $48(4.88 \%)$ & $201(20.57 \%)$ \\
\hline Sistan and Baluchestan & $-514(-17.55 \%)$ & $-1,379(-47.09 \%)$ & $351(11.99 \%)$ \\
\hline Tehran & $3,255(18.37 \%)$ & $2,372(13.39 \%)$ & $4,137(23.35 \%)$ \\
\hline Yazd & $330(22.07 \%)$ & $200(13.35 \%)$ & $461(30.79 \%)$ \\
\hline Zanjan & $290(18.27 \%)$ & $150(9.45 \%)$ & $429(27.09 \%)$ \\
\hline Iran & $16,512(14.96 \%)$ & $12,333(11.17 \%)$ & $20,691(18.74 \%)$ \\
\hline
\end{tabular}

Note: Bold fonts illustrates the provinces with excess deaths which are statistically higher than zero.

Our estimated excess deaths for the spring of $1399 \mathrm{SH}$ are generally lower than the percentage of change in the number of deaths compared to the previous year (1398 SH). This is because of our analytic approach which considers the usual increasing trend in the annual number of deaths.

The data on incident cases of COVID-19 at the provincial level are not publicly available for this period, as MoHME only reports the provinces divided into 3 strata of high, medium, or low risk for the COVID-19 epidemic status since the first month of the spring. The data on confirmed COVID-19 deaths at the provincial level are not publicly available either and we found only one recently published report that does not cover the spring (8). Regarding other types of data on the size of the epidemic, we only found a prevalence survey using antibody tests that does not completely cover the spring, is not based on random sampling, and does not include all the provinces (9). In the absence of any official report at the provincial level for the number of cases or deaths, we used a heat map on daily hospitalization rates of COVID-19 (per 100000 individuals) in provinces, released by the head of the Public Relations and Information Center of the MoHME on Twitter (10) to explore possible correlations between our estimations of all-cause excess deaths and available data on the size of the epidemic at the provincial level. We used Pillow (11) and NumPy (12) libraries of the Python programming language (13) for image processing and numerical operations, respectively, to back-calculate the daily and cumulative hospitalization rates for each province in the spring. Figure 3 shows the correlation between provincial excess death rates and COVID-19 cumulative hospitalization rates per 100000 individuals in the spring of $1399 \mathrm{SH}$.

Like the winter of $1398 \mathrm{SH}$, we think that most of the excess deaths of the spring of $1399 \mathrm{SH}$ are related to COVID-19. We did not find any other specific reason to explain the extra deaths in the spring. The ratio of excess deaths to observed deaths was higher in the spring of 1399 $\mathrm{SH}$ compared to the winter of $1398 \mathrm{SH}$. The official data from MoHME and NOCR show $1.21 \%$ and $7.45 \%$ of the total deaths have been due to COVID-19 in the winter of $1398 \mathrm{SH}$ and the spring of $1399 \mathrm{SH}$, respectively; our estimations show $4.90 \%$ of the total deaths in winter and $14.96 \%$ of the total deaths in the spring are above the expected seasonal baseline (ie, excess deaths). This increase would not be surprising if we accept that most of the excess deaths are related to COVID-19, because the epidemic in Iran was officially started on February 19, 2020, and there was only one month of overlap between the epidemic and the winter. Many of the provinces were in the early stages of the epidemic during the last month of the winter. Also, as we discussed in our previous report, there might be a higher than the usual number of delayed registration of deaths at NOCR, because of the epidemic-related specific situation of the country (2). The gap between our estimates of excess deaths and the official number of COVID-19 deaths have become closer from more than 4 times in the winter to less than 2 times in the spring. This is also not surprising as the testing capacity was increased in the 


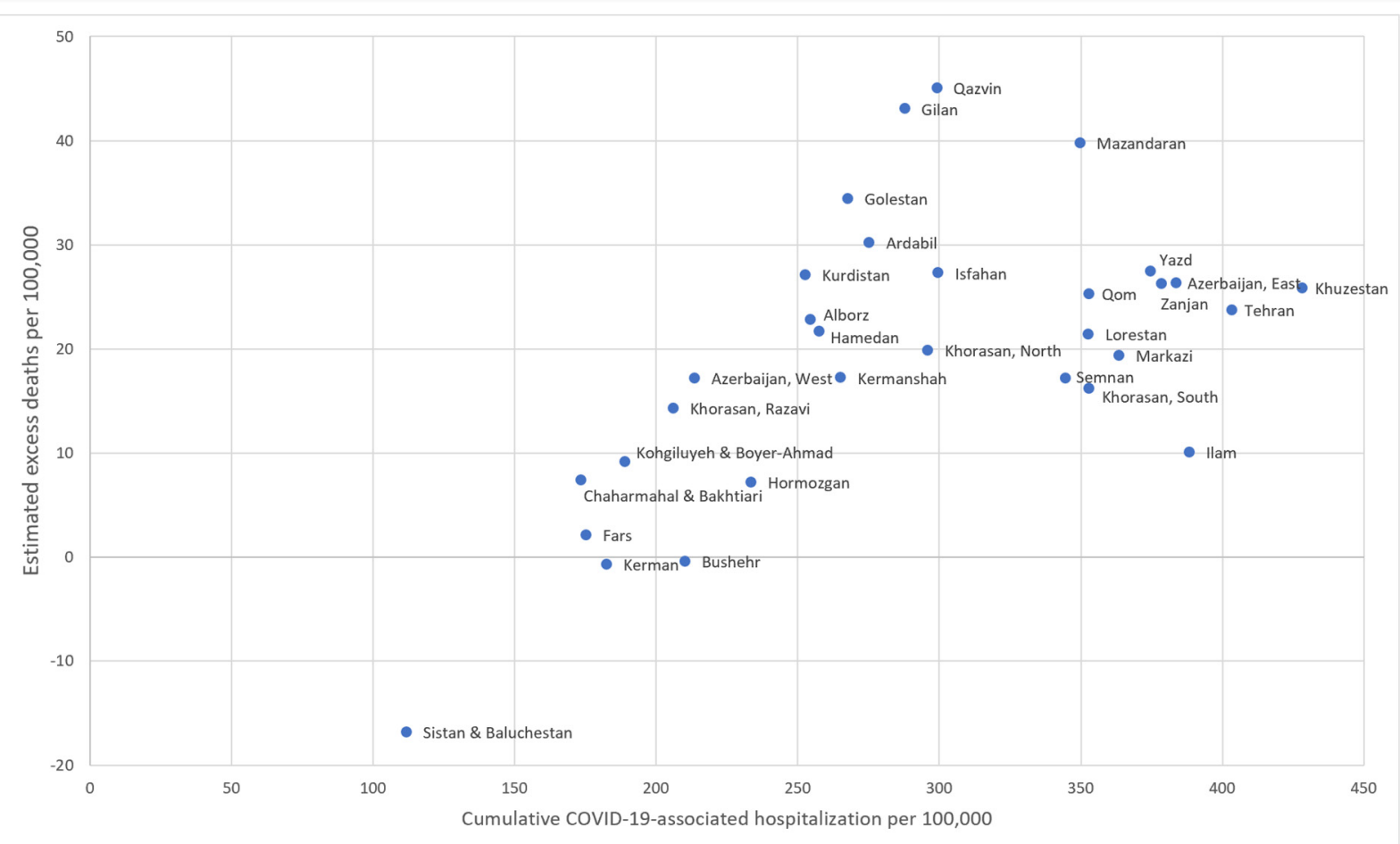

Fig. 3. Association of estimated excess deaths in the spring of 1399 SH (March 20, 2020 - June 20, 2020) and reported COVID-19 hospitalization rates among provinces of Iran

spring compared to the early days of the COVID-19 pandemic. Also, the excess deaths of winter might be a coproduct of influenza and COVID-19 epidemic, while the influenza epidemic did not continue in the spring of $1399 \mathrm{SH}$. Finally, the COVID-19 epidemic might have started sooner than the first official report on February 19, 2020; this means potential COVID-19 deaths before that date have not been included in the official reports of winter while they have affected the excess deaths.

Control of an epidemic of this magnitude is beyond the capacity of the health system and needs intersectoral collaboration and community participation. Other sectors and the general public need to be communicated regularly about their local epidemic situation, as well as national status. To facilitate monitoring of the epidemic situation, we believe that MoHME should increase public access to provincial data on the size of epidemics (such as COVID-19 deaths and cases) and resource use (such as hospitalization and number of tests performed).

\section{Acknowledgement}

We greatly appreciate Prof. Kiumarss Nasseri (Emeritus Professor of Epidemiology, School of Public Health, Tehran University of Medical Sciences) for reviewing the manuscript and providing constructive suggestions.

\section{Conflict of Interests}

The authors declare that they have no competing interests.

\section{References}

1. National Academies of Sciences, Engineering, Medicine. Evaluating Data Types: A Guide for Decision Makers using Data to Understand the Extent and Spread of COVID-19. Washington, DC: The National Academies Press; 2020.

2. Tadbiri H, Moradi-Lakeh M, Naghavi M. All-cause excess mortality and COVID-19-related deaths in Iran. Med J Islam Repub Iran. 2020;34(1):561-566.

3. World Health Organization (WHO). Coronavirus disease 2019 (COVID-19): situation report, 60. 2020 March 19. Available from: https://www.who.int/docs/default-source/coronaviruse/situation-rep orts/20200320-sitrep-60-covid-19.pdf.

4. World Health Organization (WHO). Coronavirus disease 2019 (COVID-19): situation report, 153. 2020 June 21. Available from: https://www.who.int/docs/default-source/coronaviruse/situation-rep orts/20200621-covid-19-sitrep-153.pdf.

5. National Organization for Civil Registration (NOCR). Registered Deaths by Province by Sex. Tehran, Iran: 2020 January 20. [updated 2020 June 29; cited 2020 June 29]. Available from: https://www.sabteahval.ir/avej/Page.aspx?mId=49826\&ID=2182\&Pag $\mathrm{e}=$ Magazines/SquareshowMagazine. Persian.

6. Marti-Soler H, Gonseth S, Gubelmann C, Stringhini S, Bovet P, Chen $\mathrm{PC}$, et al. Seasonal variation of overall and cardiovascular mortality: a study in 19 countries from different geographic locations. PLoS One. 2014;9(11):e113500.

7. Muscatello DJ, Cretikos MA, MacIntyre CR. All-cause mortality during first wave of pandemic (H1N1) 2009, New South Wales, Australia, 2009. Emerg Infect Dis. 2010;16(9):1396.

8. Peykari N, Mostafavi E, Eybpoosh S, Sharifi H, Haghdoost A. Trend of the COVID-19 Pandemic in Iran. Iran J Culture Health Prom. 2020;4(1):14-19. (Persian)

9. COVID-19 Epidemiological Analysis Committee of MoHME. Analysis of the latest seroepidemiological findings of COVID-19. 2020 May 15. Available from:

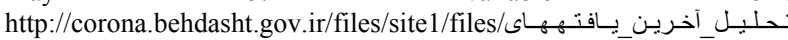
19 ــــ_pdersian.

10. Jahanpur K. Changes in \#COVID19 status in provinces [Tweet]. Twitter; 2020 July 24. [cited 2020 July 27]. Available from: https://twitter.com/drjahanpur/status/1286748079916539908. Persian.

11. Clark A, Contributors. Pillow (PIL Fork) documentation. Version 7.2.0. 2020. Available from: https://pillow.readthedocs.io/en/ stable/reference/.

12. Oliphant TE, Contributors. NumPy Reference. Version 1.19. 2020. Available from: https://numpy.org/doc/1.19/reference/. 
13. Python Software Foundation. The Python Language Reference. Version 3.8.5. 2019. Available from: https://docs.python.org /3.8/reference/. 